\title{
Epidemiology of Influenza
}

\author{
Jian Wang
}

\subsection{Source of Infection}

different subtypes of type A influenza virus. And influenza virus may invade an individual repeatedly.

The patients with influenza or latent infection are the main source of its infection, and the infectivity persists for 1-7 days after onset, which is the strongest during the first 2-3 days after onset. Pigs, cattles, horses and other poultries may also spread influenza.

\subsection{Route of Transmission}

Influenza spreads via droplets in air containing the virus produced by coughing and sneezing of persons with infection. The virus can survive in the air for about half an hour. Healthy individuals can be infected via contacts of hands to mouth or nose following contacts to utensils carrying the virus.

\subsection{Susceptible Population}

People are generally susceptible to influenza, and certain immunity can be acquired after infection. No cross immunity exists among the three types of influenza virus and among

J. Wang

Affiliated First Hospital, Xinjiang Medical University, Urumuqi, Xinjiang, China

e-mail: jeanw1265@163.com

\subsection{Prevalence}

Influenza occurs suddenly with rapid spread, and its prevalence peaks within 2-3 weeks. Its incidence is high, but only prevails in a short period of time, commonly 6-8 weeks. It commonly spreads along transportation routes, from city to countryside and from working unit to residential area. Influenza occurs all year round, and more commonly in winters and springs. In southern China, its prevalence occurs also in summers and autumns. A small scale prevalence of influenza caused by type A influenza virus occurs every 2-3 years, and a large scale prevalence every $10-15$ years. Concerning influenza caused by type B influenza virus, outbreaks or small scale prevalence occur. As for influenza caused by type $\mathrm{C}$ influenza virus, its occurrence is sporadic. 